\title{
Assessing Nonlinear Granger Causality from Multivariate Time Series
}

\author{
Xiaohai Sun \\ Max Planck Institute for Biological Cybernetics \\ Spemannstr. 38, 72076 Tübingen, Germany \\ xiaohai.sun@tuebingen.mpg.de
}

\begin{abstract}
A straightforward nonlinear extension of Granger's concept of causality in the kernel framework is suggested. The kernel-based approach to assessing nonlinear Granger causality in multivariate time series enables us to determine, in a model-free way, whether the causal relation between two time series is present or not and whether it is direct or mediated by other processes. The trace norm of the so-called covariance operator in feature space is used to measure the prediction error. Relying on this measure, we test the improvement of predictability between time series by subsampling-based multiple testing. The distributional properties of the resulting p-values reveal the direction of Granger causality. Experiments with simulated and real-world data show that our method provides encouraging results.
\end{abstract}

Keywords: time series, Granger causality, kernel methods.

\section{Introduction}

In this paper, a time series $\mathrm{X}:=\left(\ldots, x_{t-1}, x_{t}, x_{t+1}, \ldots\right)$ is a discrete time, continuous state process where $t \in \mathbb{Z}$ is a certain discrete time point. Time points are usually taken at equally spaced intervals. We consider, without loss of generality, a triviariate time series $(\mathrm{X}, \mathrm{Y}, \mathrm{Z})$ measured simultaneously, where $\mathrm{X}, \mathrm{Y}, \mathrm{Z}$ can, in general, again be multivariate time series. The problem that we focus on is that whether the underlying process of $\mathrm{X}$ is causal to the underlying process of $\mathrm{Y}$ and/or the other way around, when the underlying process $\mathrm{Z}$ is known. The well-known concept of causality in analysis of times series is the so-called Granger causality: The process $\mathrm{X}$ Granger causes another process $\mathrm{Y}$ given a third process $\mathrm{Z}$ (subsequently denoted as $\mathrm{X} \Rightarrow \mathrm{Y} \mid \mathrm{Z}$ ), if future values of $\mathrm{Y}$ can be better predicted using the past values of $(\mathrm{X}, \mathrm{Y}, \mathrm{Z})$ compared to using the past values of $(Y, Z)$ alone.

For the sake of simplicity, we first start with the bivariate case (X, Y). The time-delayed embedding vector reconstructing the state (or phase) space of times series $\mathrm{X}$ is expressed as

$$
X_{t, r, m}=\left(x_{t-(m-1) r}, \ldots, x_{t-2 r}, x_{t-r}, x_{t}\right)^{T},
$$


where $m$ is the embedding dimension and $r$ is the time delay (or lag) between successive elements of the state vector [12. The choice of $m, r$ depends on the dynamics of underlying process. If not otherwise stated in this paper, $r=1$, $m=100$ and the expression $X_{t}=\left(x_{t-99}, \ldots, x_{t-2}, x_{t-1}, x_{t}\right)^{T}$ is used. $Y_{t}$ can be defined analogously. For a more principled choice of the embedding dimension and the time lag we refer to 34. Like many of the existing causality measures, the test described in this paper is also based on the notion of the embedding vector. To assess the causal hypothesis $\mathrm{X} \Rightarrow \mathrm{Y}$, Granger causality considers the following autoregressive predictions:

$$
Y_{t+1}=a^{T} X_{t}+\epsilon_{t}^{(\mathrm{Y})} \quad \text { and } \quad Y_{t+1}=b_{1}^{T} Y_{t}+b_{2}^{T} X_{t}+\epsilon_{t}^{(\mathrm{Y} \mid X)}
$$

where $\epsilon_{t}^{(\mathrm{Y})}$ and $\epsilon_{t}^{(\mathrm{Y} \mid \mathrm{X})}$ represent the prediction errors, and $a, b_{1}, b_{2}$ denote regression coefficient vectors. The coefficient vectors and constants are determined so as to minimize the variance of $\epsilon_{t}^{(\mathrm{Y})}$ and $\epsilon_{t}^{(\mathrm{Y} \mid \mathrm{X})}$. Once the coefficient vectors have been calculated, the causal inference of $\mathrm{X}$ on $\mathrm{Y}$ can be verified if the variance $\operatorname{Var}\left[\epsilon_{t}^{(\mathrm{Y} \mid \mathrm{X})}\right]$ is significantly smaller than $\operatorname{Var}\left[\epsilon_{t}^{(\mathrm{Y})}\right]$. The opposite direction $\mathrm{X} \Leftarrow \mathrm{Y}$ can be analogously tested. Such standard test of Granger causality developed by Granger [5] assumes linear regression models, and its application to nonlinear systems may not be appropriate in the general case. A nonlinear extension of Granger causality, called the extended Granger causality index (EGCI) was proposed in [6]. The main idea of this technique is to divide the phase space into a set of small neighborhoods and approximate the globally nonlinear dynamics by local linear regression models. However, the local linearity is obviously a restrictive assumption. Another recently introduced nonlinear extensions of Granger causality (NLGC) [7] is based on kernel autoregression scheme, instead of a linear autoregression. Although this method takes no restrictive assumption of linearity, the method is fixed to a certain class of functions, i.e., nonlinear radial based functions (RBFs) and assume the additivity of the interaction between these functions. In this paper, we generalize the Granger causality in the kernel framework and present a straightforward nonlinear extension.

\section{Kernel Framework}

A positive definite kernel $k_{\mathcal{X}}: \mathcal{X} \times \mathcal{X} \rightarrow \mathbb{R}$ on a non-empty set $\mathcal{X}$ is a symmetric function, i.e., $k_{\mathcal{X}}\left(x, x^{\prime}\right)=k_{\mathcal{X}}\left(x^{\prime}, x\right)$ for any $x, x^{\prime} \in \mathcal{X}$ such that for arbitrary $n \in \mathbb{N}$ and $x^{(1)}, \ldots, x^{(n)} \in \mathcal{X}$ the matrix $K$ with $(K)_{i j}:=k_{\mathcal{X}}\left(x^{(i)}, x^{(j)}\right)$ is positive definite, i.e., $\sum_{i, j=1}^{n} c_{i} c_{j} k_{\mathcal{X}}\left(x^{(i)}, x^{(j)}\right) \geq 0$ for all $c_{1}, \ldots, c_{n} \in \mathbb{R}$. A reproducing kernel Hilbert space (RKHS) [8] $\mathcal{H}_{\mathcal{X}}$ is a Hilbert space defined by the completion of an inner product space of functions $k_{\mathcal{X}}(x, \cdot)$ with $x \in \mathcal{X}$ and the inner product defined by $\left\langle k_{\mathcal{X}}(x, \cdot), k_{\mathcal{X}}\left(x^{\prime}, \cdot\right)\right\rangle=k_{\mathcal{X}}\left(x, x^{\prime}\right)$ for all $x, x^{\prime} \in \mathcal{X}$. In other words, $\Phi(x)(\cdot)=k_{\mathcal{X}}(x, \cdot)$ defines a map from $\mathcal{X}$ into a feature space $\mathcal{H}_{\mathcal{X}}$. With the so-called "kernel trick", a linear algorithm can easily be transformed into a non-linear algorithm, which is equivalent to the linear algorithm operating in the space of $\Phi$. However, because the kernel function is used for calculating 
the inner product, the mapping $\Phi$ is never explicitly computed. This is desirable, because the high-dimensional space may be infinite-dimensional, as is the case when the kernel is ,e.g., a Gaussian or Laplacian.

In kernel framework, the autoregression models of Eq. 2 can be replaced by

$$
a^{T} \Phi\left(Y_{t+1}\right)=b_{1}^{T} \Psi\left(Y_{t}\right)+\epsilon_{t}^{(\mathrm{Y})} \quad \text { and } \quad a^{T} \Phi\left(Y_{t+1}\right)=b_{2}^{T} \Psi\left(Y_{t}, X_{t}\right)+\epsilon_{t}^{(\mathrm{Y} \mid \mathrm{X})}
$$

where $\Phi, \Psi$ are nonlinear maps into feature spaces $\mathcal{H}_{\mathcal{Y}}, \mathcal{H}_{\mathcal{Y} \otimes \mathcal{X}}$ respectively. In the conditional case (conditioning on $\mathrm{Z}$ ), we will have

$$
a^{T} \Phi\left(Y_{t+1}\right)=b_{1}^{T} \Psi\left(Y_{t}, Z_{t}\right)+\epsilon_{t}^{(\mathrm{Y})} \text { and } a^{T} \Phi\left(Y_{t+1}\right)=b_{2}^{T} \Psi\left(Y_{t}, Z_{t}, X_{t}\right)+\epsilon_{t}^{(\mathrm{Y} \mid \mathrm{X})}
$$

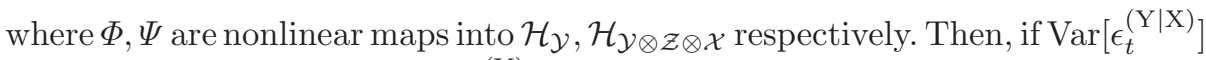
is significant smaller than $\operatorname{Var}\left[\epsilon_{t}^{(\mathrm{Y})}\right], \mathrm{X} \Rightarrow \mathrm{Y} \mid \mathrm{Z}$ can be verified, otherwise no evidence for Granger causality. To test this significance, we define the covariance operator 9] expressing variance of variables in the feature space.

\subsection{Covariance Operator}

Suppose we have random vector $(X, Y)$ taking values on $\mathcal{X} \times \mathcal{Y}$. The base spaces $\mathcal{X}$ and $\mathcal{Y}$ are topological spaces. Measurability of these spaces is defined with respect to the Borel $\sigma$-field. The joint distribution of $(X, Y)$ is denoted by $P_{X Y}$ and the marginal distributions by $P_{X}$ and $P_{Y}$. Let $\left(\mathcal{X}, \mathcal{B}_{\mathcal{X}}\right)$ and $\left(\mathcal{Y}, \mathcal{B}_{\mathcal{Y}}\right)$ be measurable spaces and $\left(\mathcal{H}_{\mathcal{X}}, k_{\mathcal{X}}\right),\left(\mathcal{H}_{\mathcal{Y}}, k_{\mathcal{Y}}\right)$ be RKHSs of functions on $\mathcal{X}$ and $\mathcal{Y}$ with positive definite kernels $k_{\mathcal{X}}, k_{\mathcal{Y}}$. We consider only random vectors $(X, Y)$ on $\mathcal{X} \times \mathcal{Y}$ such that the expectations $\mathrm{E}_{X}\left[k_{\mathcal{X}}(X, X)\right], \mathrm{E}_{Y}\left[k_{\mathcal{Y}}(Y, Y)\right]$ are finite, which guarantees $\mathcal{H}_{\mathcal{X}}$ and $\mathcal{H}_{\mathcal{Y}}$ are included in $L^{2}\left(P_{X}\right)$ and $L^{2}\left(P_{Y}\right)$ respectively, where $L^{2}(\mu)$ denotes the Hilbert space of square integrable functions with respect to a measure $\mu$. It is known that there exists a unique operator $\Sigma_{Y X}$, called crosscovariance operator, from $\mathcal{H}_{\mathcal{X}}$ to $\mathcal{H}_{\mathcal{Y}}$ such that

$$
\left\langle g, \Sigma_{Y X} f\right\rangle_{\mathcal{H}_{\mathcal{Y}}}=\mathrm{E}_{X Y}[f(X) g(Y)]-\mathrm{E}_{X}[f(X)] \mathrm{E}_{Y}[g(Y)]=\operatorname{Cov}[f(X), g(Y)]
$$

for all $f \in \mathcal{H}_{\mathcal{X}}, g \in \mathcal{H}_{\mathcal{Y}}$. Here, $\operatorname{Cov}[\cdot]$ denotes the covariance. $\mathrm{E}_{X}[\cdot], \mathrm{E}_{Y}[\cdot]$ and $\mathrm{E}_{X Y}[\cdot]$ denote the expectation over $P_{X}, P_{Y}$ and $P_{X Y}$, respectively. Baker [9] showed that $\Sigma_{Y X}$ has a representation of the form $\Sigma_{Y X}=\Sigma_{Y Y}^{1 / 2} V_{Y X} \Sigma_{X X}^{1 / 2}$ with a unique bounded operator $V_{Y X}: \mathcal{H}_{\mathcal{X}} \rightarrow \mathcal{H}_{\mathcal{Y}}$ such that $\left\|V_{Y X}\right\| \leq 1$, where $\|\cdot\|$ is used for the operator norm of a bounded operator, i.e., $\|V\|=\sup _{\|f\|=1}\|V f\|$. Moreover, it is obvious that $\Sigma_{X Y}=\Sigma_{Y X}^{*}$, where $\Sigma^{*}$ denotes the adjoint of an operator $\Sigma$. If $X$ is equal to $Y$, the positive self-adjoint operator $\Sigma_{Y Y}$ is called the covariance operator.

Based on the cross-covariance operator, we introduce the conditional covariance operator. Let $\left(\mathcal{H}_{\mathcal{S}}, k_{\mathcal{S}}\right),\left(\mathcal{H}_{\mathcal{T}}, k_{\mathcal{T}}\right),\left(\mathcal{H}_{\mathcal{Y}}, k_{\mathcal{Y}}\right)$ be RKHSs on measurable spaces $\mathcal{S}, \mathcal{T}, \mathcal{Y}$ respectively. Let $(X, Y)=(S, T, Y)$ be a random vector on $\mathcal{S} \times \mathcal{T} \times \mathcal{Y}$, where $X=(S, T)$ and $\mathcal{H}_{\mathcal{X}}=\mathcal{H}_{\mathcal{S}} \otimes \mathcal{H}_{\mathcal{T}}$. The positive self-adjoint operator $\Sigma_{Y Y \mid X}:=\Sigma_{Y Y}-\Sigma_{Y Y}^{1 / 2} V_{Y X} V_{X Y} \Sigma_{Y Y}^{1 / 2}$ is called the conditional covariance operator, 
where $V_{Y X}$ and $V_{X Y}$ are the bounded operators derived from $\Sigma_{Y X}$ and $\Sigma_{X Y}$. If $\Sigma_{X X}^{-1}$ exists, we can rewrite $\Sigma_{Y Y \mid X}$ as $\Sigma_{Y Y \mid X}=\Sigma_{Y Y}-\Sigma_{Y X} \Sigma_{X X}^{-1} \Sigma_{X Y}$. Fukumizu et al. 10, Proposition 2] showed that $\left\langle g, \Sigma_{Y Y \mid X} g\right\rangle_{\mathcal{H}_{Y}}=\inf _{f \in \mathcal{H}_{X}} \mathrm{E}_{X Y}[\mid(g(Y)-$ $\left.\left.\mathrm{E}_{Y}[g(Y)]\right)-\left.\left(f(X)-\mathrm{E}_{X}[f(X)]\right)\right|^{2}\right]$ for any $g \in \mathcal{H}_{\mathcal{Y}}$. This is an analogous to the well-known results on covariance matrices and linear regression: The conditional covariance matrix $C_{Y Y \mid X}=C_{Y Y}-C_{Y X} C_{X X}^{-1} C_{X Y}$ expresses the residual error of the least square regression problem as $b^{\mathrm{T}} C_{Y Y \mid X} b=\min _{a} \mathrm{E}_{X Y}\left\|b^{\mathrm{T}} Y-a^{\mathrm{T}} X\right\|^{2}$. To relate this residual error to the conditional variance of $g(Y)$ given $X$, the following assumption for RKHSs is made.

Assumption 1. Let $\left(\mathcal{H}_{\mathcal{X}}, k_{\mathcal{X}}\right)$ be an RKHS on measurable space $\mathcal{X}$, then $\mathcal{H}_{\mathcal{X}}+\mathbb{R}$ is dense in $L^{2}\left(P_{X}\right)$ for every probability measure $P_{X}$ on $\mathcal{X}$, where " + " means the direct sum of Hilbert spaces.

This assumption is the necessary and sufficient condition that the kernels are "characteristic". The notation of the characteristic kernels is a generalization of the characteristic function $\mathrm{E}_{X}\left[\exp \left(\sqrt{-1} u^{\mathrm{T}} X\right)\right]$, which is the expectation of the (complex-valued) positive definite kernel $k(x, u)=\exp \left(\sqrt{-1} u^{\mathrm{T}} x\right.$ ) (see 11] for details). One popular class of characteristic kernels is the universal kernels [12] on a compact metric space, e.g., the Gaussian RBF kernel or Laplacian kernel on a compact subset of $\mathbb{R}^{m}$, because the Banach space of bounded continuous functions on a compact subset $\mathcal{X}$ of $\mathbb{R}^{m}$ is dense in $L^{2}\left(P_{X}\right)$ for any $P_{X}$ on $\mathcal{X}$. Another example is the Gaussian or Laplacian kernel on the entire Euclidean space, since many random variables are defined on non-compact spaces. One can prove that, Assumption 11holds for these kernels (see Appendix A1 in 13 for the proof). Under Assumption 1, one can show that $\left\langle g, \Sigma_{Y Y \mid X} g\right\rangle_{\mathcal{H}_{\mathcal{Y}}}=\mathrm{E}_{X}\left[\operatorname{Var}_{Y \mid X}[g(Y) \mid X]\right]$ for all $g \in \mathcal{H}_{\mathcal{Y}}$. Thus, the conditional covariance operator expresses the conditional variance of $g(Y)$ given $X$ in the feature space. As a side note, Ancona et al. 14 claimed that not all kernels are suitable for their nonlinear prediction schemes. They presented only sufficient conditions, which hold for Gaussian kernels. The kernel framework allows wider class of kernels and the kernel functions for $k_{\mathcal{X}}$

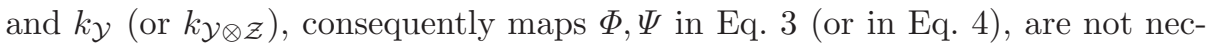
essarily to belong to the same function class. In particular, even though one can overall use Gaussian kernels $k_{\mathcal{X}}: \mathbb{R}^{m} \times \mathbb{R}^{m} \rightarrow \mathbb{R}, k_{\mathcal{X}}\left(x, x^{\prime}\right)=\exp \left(-\left\|x-x^{\prime}\right\|^{2} / 2 \sigma^{2}\right)$ (as we did in all experiments), the parameter $\sigma^{2}$ of kernels can be adapted to different variables independently. We set the parameter such that $2 \sigma^{2}$ equals the variance of the variable. For these reasons, our framework is more general and flexible than the framework of the state-of-the-art approaches, as described in 6]7]15.

To evaluate the conditional covariance operator, we use the trace norm, because it is not difficult to see that the trace norm of the operator is directly linked with the sum of residual errors, namely

$$
\operatorname{Tr}\left(\Sigma_{Y Y \mid X}\right)=\sum_{i} \min _{f \in \mathcal{H}_{X}} \mathrm{E}_{X Y}\left[\left|\left(\phi_{i}(Y)-\mathrm{E}_{Y}\left[\phi_{i}(Y)\right]\right)-\left(f(X)-\mathrm{E}_{X}[f(X)]\right)\right|^{2}\right],
$$

where $\left\{\phi_{i}\right\}_{i=1}^{\infty}$ is the complete orthonormal system of the separable RKHS $\mathcal{H}_{\mathcal{Y}}$. An RKHS $\left(\mathcal{H}_{\mathcal{Y}}, k_{\mathcal{Y}}\right)$ is separable, when the topological space $\mathcal{Y}$ is separable 
and $k_{\mathcal{Y}}$ is continuous on $\mathcal{Y} \times \mathcal{Y}$ [16]. It can be shown that $\Sigma_{Y Y \mid X} \leq \Sigma_{Y Y \mid S}$ with $X=(S, T)$, where the inequality refers to the usual order of self-adjoint operators, namely if $A \leq B \Leftrightarrow\langle A g, g\rangle \leq\langle B g, g\rangle$ for all $g \in \mathcal{H}_{\mathcal{Y}}$. Further, if $\mathcal{H}_{\mathcal{X}}, \mathcal{H}_{\mathcal{Y}}, \mathcal{H}_{\mathcal{S}}$ are given by characteristic kernels, $\Sigma_{Y Y \mid X}=\Sigma_{Y Y \mid S} \Leftrightarrow Y \Perp T \mid S$, which denotes that $Y$ and $T$ are conditionally independent, given $S$ (denoted by $Y \Perp T \mid S$ ). In terms of the trace norm, we have the following property:

Property 1. Let $\mathbb{T}_{Y Y \mid X}$ denote the trace norm of the conditional covariance operator $\operatorname{Tr}\left(\Sigma_{Y Y \mid X}\right)$ with $X:=(S, T)$. Then we have $\mathbb{T}_{Y Y \mid S, T}<\mathbb{T}_{Y Y \mid S} \Leftrightarrow Y \not 1$ $T \mid S$ and $\mathbb{T}_{Y Y \mid S, T}=\mathbb{T}_{Y Y \mid S} \Leftrightarrow Y \Perp T \mid S$.

All technical details are omitted due to space limitations. Property 1 generalizes the (P1)-property, required by Ancona et al. [7, Section II.A] for any nonlinear extension of Granger causality, since (P1)-property describes merely the bivariate case, while Property 1 holds for multivariate cases.

\subsection{Empirical Estimation of Operators}

In analogy to [11, we introduce the estimations of $\mathbb{T}_{Y Y}$ and $\mathbb{T}_{Y Y \mid X}$ based on sample $\left(x^{(1)}, y^{(1)}\right), \ldots,\left(x^{(n)}, y^{(n)}\right)$ from the joint distribution. Using the empirical mean elements $\widehat{m}_{X}^{(n)}=\frac{1}{n} \sum_{i=1}^{n} k_{\mathcal{X}}\left(x^{(i)}, \cdot\right)$ and $\widehat{m}_{Y}^{(n)}=\frac{1}{n} \sum_{i=1}^{n} k_{\mathcal{Y}}\left(y^{(i)}, \cdot\right)$, an estimator of $\Sigma_{Y X}$ is $\widehat{\Sigma}_{Y X}^{(n)}=\frac{1}{n} \sum_{i=1}^{n}\left(k_{\mathcal{Y}}\left(y^{(i)}, \cdot\right)-\widehat{m}_{Y}^{(n)}\right)\left\langle k_{\mathcal{X}}\left(x^{(i)}, \cdot\right)-\widehat{m}_{X}^{(n)}, \cdot\right\rangle_{\mathcal{H}_{\mathcal{X}}}$. $\widehat{\Sigma}_{Y Y}^{(n)}$ and $\widehat{\Sigma}_{X X}^{(n)}$ can be defined accordingly. An estimator of $\Sigma_{Y Y \mid X}$ is then defined by $\widehat{\Sigma}_{Y Y \mid X}^{(n, \epsilon)}=\widehat{\Sigma}_{Y Y}^{(n)}-\widehat{\Sigma}_{Y X}^{(n)}\left(\widehat{\Sigma}_{X X}^{(n)}+\epsilon I\right)^{-1} \widehat{\Sigma}_{X Y}^{(n)}$, where $\epsilon>0$ is a regularization constant that enables inversion 1 It can be shown that $\widehat{\mathbb{T}}_{Y Y}^{(n)}=\operatorname{Tr}\left(\widehat{\Sigma}_{Y Y}^{(n)}\right)$ is a consistent estimator of $\mathbb{T}_{Y Y}$, which guarantees to converge in Hilbert-Schmidt norm at rate $n^{-1 / 2}$. Moreover, $\widehat{\mathbb{T}}_{Y Y \mid X}^{(n, \epsilon)}=\operatorname{Tr}\left(\widehat{\Sigma}_{Y Y \mid X}^{(n, \epsilon)}\right)$ is a consistent estimator of $\mathbb{T}_{Y Y \mid X}$. If $\epsilon$ converges to zero more slowly than $n^{-1 / 2}$, this estimator converges to $\mathbb{T}_{Y Y \mid Z}$ (see [13] for proofs). For notational convenience, we will henceforth omit the upper index and use $\widehat{\mathbb{T}}_{Y Y}$ and $\widehat{\mathbb{T}}_{Y Y \mid X}$ to denote the empirical estimators. The computation with kernel matrices of $n$ data points becomes infeasible for very large $n$. In our practical implementation, we use the incomplete Cholesky decomposition $\widehat{K}=L L^{\mathrm{T}}[19$, where $L$ is a lower triangular matrix determined uniquely by this equation. This may lead to considerably fewer columns than the original matrix. If $k$ columns are returned, the storage requirements are $O(k n)$ instead of $O\left(n^{2}\right)$, and the running time of many matrix operations reduces to $O\left(n k^{2}\right)$ instead of $O\left(n^{3}\right)$.

\footnotetext{
${ }^{1}$ The regularizer is required as the number of observed data points is finite, whereas the feature space could be infinite-dimensional. The regularization may be understood as a smoothness assumption on the eigenfunctions of $\mathcal{H}_{\mathcal{X}}$. It is analogous to Tikhonov regularization [17] or ridge regression [18. Many simulated experiments showed that the empirical measures are insensitive to $\epsilon$, if it is chosen in some appropriate interval, e.g., $\left[10^{-10}, 10^{-2}\right]$. We chose $\epsilon=10^{-5}$ in all our experiments.
} 


\section{Subsampling-Based Testing of Granger Causality}

Essentially, Granger causality is expressed in terms of predictability: If data vector $X$ causally influences data vector $Y$, then $Y$ would be predictable by $X$, we denote $X \rightarrow Y$. Shorthand $X \nrightarrow Y$ denotes the fact that the predictability on $Y$ by $X$ cannot be verified. Note that, in this paper, we distinguish between simple arrow " $\rightarrow$ " expressing predictability and the double arrow " $\Rightarrow$ " expressing Granger causality. To capture the predictability in a nonlinear setting by a significance test, we take the empirical estimation of the trace norm $\widehat{\mathbb{T}}_{Y Y \mid X}$ and conduct permutation tests to simulate this measure under the null hypothesis, i.e., unpredictable. Given a set of random permutations $\left\{\pi_{1}, \ldots, \pi_{m}\right\}$, we shuffle the data vector $X=\left(x^{(1)}, \ldots, x^{(n)}\right)^{T}$ and calculate the empirical null distribution $\left\{\widehat{\mathbb{T}}_{Y Y \mid X^{\pi_{1}}}, \ldots, \widehat{\mathbb{T}}_{Y Y \mid X^{\pi_{m}}}\right\}$. Then, we determine the p-value $p$, which is the percentage of values in $\left\{\widehat{\mathbb{T}}_{Y Y \mid X^{\pi_{1}}}, \ldots, \widehat{\mathbb{T}}_{Y Y \mid X^{\pi_{m}}}\right\}$, which are properly less than $\widehat{\mathbb{T}}_{Y Y \mid X}$. Smaller p-values suggest stronger evidence against the null hypothesis (unpredictable), and thus stronger evidence favoring the alternative hypothesis (predictable). As a cut-off point for p-values, a significance level $\alpha$ is pre-specified. A typical choice is $\alpha=0.05$. If $p<\alpha$, the null hypothesis is rejected, which means the predictability $X \rightarrow Y$ is significantly verified; Otherwise, we have $X \nrightarrow Y$. This procedure can be straightforwardly extended to testing conditional predictability, i.e., $X \rightarrow Y \mid Z$. The measure and the corresponding empirical null distribution will be $\widehat{\mathbb{T}}_{Y Y \mid Z, X}$ and $\left\{\widehat{\mathbb{T}}_{Y Y \mid Z, X^{\pi_{1}}}, \ldots, \widehat{\mathbb{T}}_{Y Y \mid Z, X^{\pi_{m}}}\right\}$. It is remarkable that the test of predictability can be straightforwardly applied to multi-dimensional conditioning variables. Such cases are usually difficult by partitioning and Parzen-window approaches, which are common practice for nonparametric conditional independence tests. $X, Y, Z$ in " $X \rightarrow Y \mid Z$ " could be usual random variables, and are not necessarily time series. The test of predictability alone does not use the temporal information. Granger's concept bases the asymmetry of causality on the time flow. Consequently, to test whether a time series $\mathrm{X}$ Granger causes another time series Y, we should first filter out the prediction power of past values of $\mathrm{Y}$. For this purpose, we expand $\mathrm{X}$ with embedding vectors $\left\{\ldots, X_{t-1}, X_{t}, X_{t+1}, \ldots\right\}$ and the system $\mathrm{Y}$ to $\left\{\ldots, Y_{t-1}, Y_{t}, Y_{t+1}, \ldots\right\}$. In doing so, we can test predictability in a temporal context.

So far, we have shown the test of predictability on a single sample. The statistical power of such single tests is limited if the number of data points involved is not very large. Although long time series are often available in real applications, working with kernel matrices is only tractable, if the number of data points is not too large. For these reasons, we propose to utilize subsampling-based multiple testing. If the subsample size is moderate, the multiple testing remains feasible in spite of replications of the single test. In particular, such a multiple test can significantly increase the power of statistical tests in comparison to a single test [20]. In addition,the subsampling-based procedure characterizes the predictability through local segments of time series, i.e., sub-time-series, which makes the test somewhat robust with respect to e.g., global non-stationarity of 
the time series. Fig. 1 summarizes the multiple testing procedure for detecting Granger causality. Step 1 runs single hypothesis tests on $N$ random subsamples and obtains $N$ p-values, one for each subsample. Based on the distributional properties of these p-values, Step 2 makes the decision on the predictability. In details, Step 1.1 searches for the smallest integer $l_{Y} \in\{0,1,2, \ldots\}$, which states that $\left\{Y_{t-l_{\mathrm{Y}}}, \ldots, Y_{t-1}\right\}$ (empty set for $l_{\mathrm{Y}}=0$ ) achieves the maximum knowledge that would be useful to predict $Y_{t}$. Step 1.2 yields a p-value which contains the information about whether $X_{t-1}$ can additionally improve the prediction of $Y_{t}$, given the maximum past knowledge of $\mathrm{Y}$. Due to the strict time order requirement of Granger's concept, the instantaneous influence within one time slice is excluded. We test the one-step-ahead prediction, because an influence from $X_{t-j}(j=2,3, \ldots)$ to $Y_{t}$ can be usually be captured by $X_{t-1}$ to $Y_{t}$, if $X_{t-j}$ is dependent of $X_{t-1}$.

To make the second step apparent, let us take a closer look at the distribution of p-values. According to Property 1 , we have $\mathbb{T}_{Y Y \mid X} \leq \mathbb{T}_{Y Y}$ and $\mathbb{T}_{Y Y \mid X^{\pi_{i}}} \leq$

Hypothesis: $\mathrm{X} \Rightarrow \mathrm{Y} \mid \mathrm{Z}$.

Step 1: For each sub-time series $\left(\mathrm{X}^{(i)}, \mathrm{Y}^{(i)}, \mathrm{Z}^{(i)}\right)(i=1, \ldots, N)$ of time series $(\mathrm{X}, \mathrm{Y}, \mathrm{Z})$ :

Step 1.1: If $\left(Y_{t-1}^{(i)}, Z_{t-1}^{(i)}\right) \nrightarrow Y_{t}^{(i)}$, set time lag $l_{\mathrm{Y}}^{(i)}=0$, else find the smallest lag $l_{\mathrm{Y}}^{(i)} \in\{1,2, \ldots\}$ such that $\left(Y_{t-l_{\mathrm{Y}-1}}^{(i)}, Z_{t-l_{\mathrm{Y}-1}}^{(i)}\right) \nrightarrow$ $Y_{t}^{(i)} \mid Y_{t-1}^{(i)}, \ldots, Y_{t-l_{\mathrm{Y}}}^{(i)}, Z_{t-1}^{(i)}, \ldots, Z_{t-l_{\mathrm{Y}}}^{(i)}$.

Step 1.2: If $l_{Y}^{(i)}=0$, test $X_{t-1}^{(i)} \rightarrow Y_{t}^{(i)}$, else test $X_{t-1}^{(i)} \rightarrow$ $Y_{t}^{(i)} \mid Y_{t-1}^{(i)}, \ldots, Y_{t-l_{\mathrm{Y}}}^{(i)}, Z_{t-1}^{(i)}, \ldots, Z_{t-l_{\mathrm{Y}}}^{(i)}$. A p-value $p_{i}$ is obtained.

Step 2: Calculate the skewness of p-values $p_{1}, \ldots, p_{N}$ and the probability $P^{\text {Unif }}$ that $p_{1}, \ldots, p_{N}$ are from a uniform distribution over $[0,1]$. If $P^{\text {Unif }}<\frac{1}{2}$ and the p-values are positively skewed, accept the hypothesis, i.e., $\mathrm{X} \Rightarrow \mathrm{Y} \mid \mathrm{Z}$; Otherwise, the hypothesis cannot be verified.

Fig. 1. Subsampling-based multiple testing of Granger causality

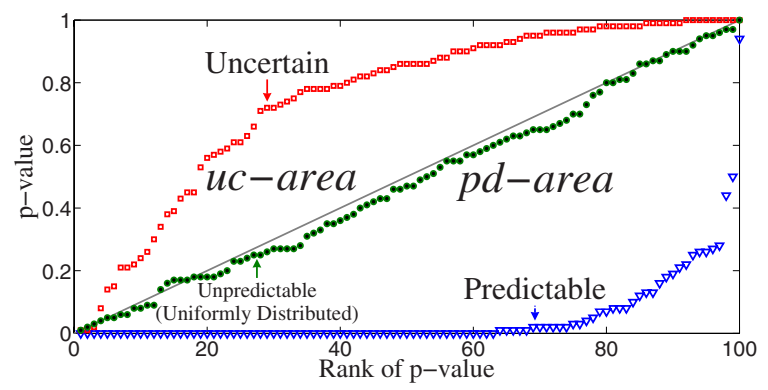

Fig. 2. Q-Q plots of p-values of three predictability tests. The field above and under the diagonal line are called uncertain (uc) area and predictable (pd) area respectively. 
$\mathbb{T}_{Y Y}$ for any permutation $\pi_{i}$. If $Y$ is predictable by $X$, we expect $\mathbb{T}_{Y Y \mid X}<$ $\mathbb{T}_{Y Y \mid X^{\pi_{i}}}$ for almost all random permutations $\pi_{i}$. Intuitively, the predictability on $Y$ by $X$ is reflected in a significant reduction of the sum of residual errors, captured by $\mathbb{T}_{Y Y \mid X}$, in comparison to $\mathbb{T}_{Y Y \mid X^{\pi_{i}}}$. Consequently, the majority of pvalues are closer to 0 and the distribution of $\mathrm{p}$-values is rather positively skewed (right-skewed). If $Y$ is unpredictable by $X$, then $\mathbb{T}_{Y Y \mid X}<\mathbb{T}_{Y Y \mid X^{\pi_{i}}}$ is as likely as $\mathbb{T}_{Y Y \mid X^{\pi_{i}}} \leq \mathbb{T}_{Y Y \mid X}$, p-values are uniformly distributed over $[0,1]$ and the skewness of p-values vanishes. If $\mathbb{T}_{Y Y \mid X^{\pi_{i}}} \leq \mathbb{T}_{Y Y \mid X}$ is true for the majority of random permutations $\pi_{i}$, more p-values are closer to 1 and the distribution of p-values is negatively skewed (left-skewed). This case, called uncertain situation, can occur due to various reasons. A very special case is, e.g., $\mathbb{T}_{Y Y \mid X}=\mathbb{T}_{Y Y \mid X^{\pi_{i}}}$, the null distribution is degenerate (all its probability mass is concentrated on one point). For instance, if $Z$ is high-dimensional or the statistical fluctuation of $Y$ is very small, it could happen that $\mathbb{T}_{Y Y \mid Z, X} \approx \mathbb{T}_{Y Y \mid Z, X^{\pi_{i}}} \approx \mathbb{T}_{Y Y \mid Z} \approx 0$. Then, the test of predictability cannot provide reliable information about the relation between $X$ and $Y$. In such cases, an uncertain situation can be interpreted as non-evidence for the hypothesis $X \rightarrow Y \mid Z$. Another interpretation of uncertain situations will be discussed later.

Inspired by 2120, we visualize these observations with an intuitive graphical tool. First, we sort the set of p-values $\left\{p_{(1)}, \ldots, p_{(N)}\right\}$ in an increasing order, i.e., $p_{1} \leq p_{2} \leq \ldots \leq p_{N}$. If $p_{i}$ behaves as an ordered sample from the uniform distribution over $[0,1]$, the expected value of $p_{i}$ is approximately $\frac{i}{N}$. The slope of $p_{i}$ versus $i$, also called Q-Q plot ("Q" stands for quantile), should exhibit a linear relationship, along a line of slope $\frac{1}{N}$ passing through the origin (diagonal line in the Q-Q plot as shown in Fig. 2). If p-values are positively skewed, the reordered p-values are located in the subfield under the diagonal line, called pd-area ("pd": predictable). If p-values are negatively skewed, the reordered p-values are located in the so-called uc-area ("uc": uncertain). For a reliable decision based on the resulting p-values, the subsamples should be independent to some degree, since independent statistics on p-values are needed. This is the case, when the given sample size is much larger than the subsample size. In all our experiments, we fixed the subsample size at 100, since time series in our experiments contain at least 5000 data points. The other parameter of the multiple testing is the number of replications of the single test: $N$. In principle, $N$ should be large enough to enable a reliable identification of uniform distribution from $N$ p-values. In our experiments, we chose $N \geq 100$. For large sample sizes, we chose $N=1000$.

The remaining problem is how to judge, in spite of the fluctuation of one specific set of p-values, whether the $N$ resulting p-values are uniformly distributed or not. We transform this problem to a two-sample-problem. More precisely, we simulate 1000 samples of $N$ values from the uniform distribution over $[0,1]$. For each of the 1000 simulated samples, we test whether the $N$ resulting p-values are identically distributed with the $N$ values from truly uniform distribution. The percentage of the positive results, i.e., the resulting p-values and the simulated values come from the same distribution, can be considered as the probability that the resulting $\mathrm{p}$-values are uniformly distributed: $P^{\mathrm{Unif}}$. If $P^{\mathrm{Unif}}<\frac{1}{2}$, the $\mathrm{p}$-values 
are less likely from a uniform distribution than from a non-uniform distribution. In our experiments, we employ the kernel-based test for the two-sample-problem proposed by Gretton et al. 222. After all, the decision of predictability relies on whether $P^{\text {Unif }}<\frac{1}{2}$ and whether the p-values are positively skewed.

\section{Experiments}

To demonstrate the effectiveness of the proposed approach, we test our method on simulated data generated by chaotic maps and benchmark data of real-life systems of different scientific fields.

\subsection{Hénon Maps}

As the first simulated example, we consider the following two noisy Hénon maps:

$$
\begin{aligned}
& x_{t+1}=a+c_{1} x_{t-1}-d_{1} x_{t}^{2}+\mu \xi_{1} \\
& y_{t+1}=a+c_{2} y_{t-1}-b x_{t} y_{t}-(1-b) d_{2} y_{t}^{2}+\mu \xi_{2}
\end{aligned}
$$

represented as systems $\mathrm{X}$ and $\mathrm{Y}$, respectively. Here, system $\mathrm{X}$ drives system $\mathrm{Y}$ with coupling strength $b \in[0,1]$. If $b=0, \mathrm{X}$ and $\mathrm{Y}$ are uncoupled; If $b>0$, we have a uni-directed coupling $\mathrm{X} \Rightarrow \mathrm{Y}$. This example is also discussed by Bhattacharya et al. 23], who proposed to choose $b<0.7$ to avoid strong synchronization. Similar to 23, we fixed the parameters at $a=1.4, c_{1}=0.3, c_{2}=0.1, d_{1}=1$, $d_{2}=0.4, \mu=0.01 . \xi_{1,2}$ are unit variance Gaussian distributed noise terms. Note that $X$ and $Y$ are different systems even in the case of $b=0$, because $c_{1} \neq c_{2}$ and $d_{1} \neq d_{2}$. Therefore, identical synchronization is impossible. We start with points $x_{1,2}=y_{1,2}=0$. The upper line of Fig. 3 shows the time series of 10000 data points. We ran our test procedure on uncoupled time series $(b=0)$ and weakly unidirectionally coupled time series $(b=0.25)$. The reordered p-values obtained in both cases are visualized in the lower line of Fig. 3. In the case of $b=0$, our test rejected the Granger causality in both directions. In the case of $b=0.25$, our test revealed $\mathrm{X} \Rightarrow \mathrm{Y}$ and gained no evidence for $\mathrm{X} \Leftarrow \mathrm{Y}$. Actually, the reordered $\mathrm{p}$ values of testing $Y_{t-1} \rightarrow X_{t} \mid X_{t-1}, \ldots, X_{t-l_{X}}$ are located in the uc-area. Based on the underlying model, the past values of $X$ can, in fact, improve the prediction of the future of $Y$. At the same time, values of $Y$ in the future (random permutation of $Y_{t-1}$ might yield that) could be helpful for guessing the past of $\mathrm{X}$. If this effect

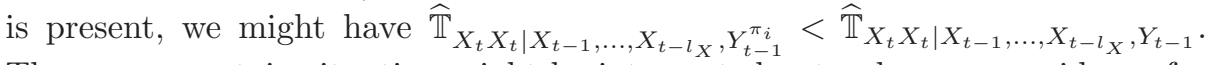
Thus, an uncertain situation might be interpreted not only as non-evidence for the direction $\mathrm{X} \Leftarrow \mathrm{Y}$ that is just tested, but also as indirect evidence for the reversed direction $\mathrm{X} \Rightarrow \mathrm{Y}$.

\subsection{Logistic Maps}

As a second example, we consider the following pair of noisy logistic maps:

$$
\begin{aligned}
& x_{t+1}=\left(1-b_{1}\right) a x_{t}\left(1-x_{t}\right)+b_{1} a y_{t}\left(1-y_{t}\right)+\mu \xi_{1} \\
& y_{t+1}=\left(1-b_{2}\right) a y_{t}\left(1-y_{t}\right)+b_{2} a x_{t}\left(1-x_{t}\right)+\mu \xi_{2}
\end{aligned}
$$



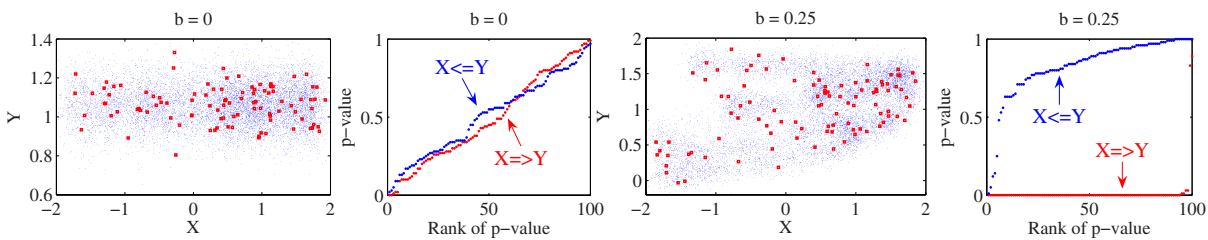

Fig. 3. The first and third plot from left show bivariate time series of 10000 observations (fine points) generated by Hénon maps and one random sub-time-series of 100 observations (bold points). The second and forth plot from left shows the corresponding Q-Q plots of p-values obtained by tests of predictability on 100 sub-time-series.

represented as systems $\mathrm{X}$ and $\mathrm{Y} . b_{1,2} \in[0,1]$ describe the coupling strengths between $\mathrm{X}$ and $\mathrm{Y}$. If $b_{1,2}=0, \mathrm{X}$ and $\mathrm{Y}$ are uncoupled; If $b_{1,2}>0$, we have a bi-directed coupling $\mathrm{X} \Leftrightarrow \mathrm{Y}$; If $b_{1}=0$ and $b_{2}>0$, we have a uni-directed coupling $\mathrm{X} \Rightarrow \mathrm{Y}$. The last case is also studied by Ancona et al. 77. They claimed that in the noise-free case, i.e., $\mu=0$, a transition to synchronization occurs at $b_{2}=0.37$ based on the calculation of the Lyapunov exponents. For this reason, we chose $b_{1,2}<0.37$. As proposed in [7, parameter $a$ is fixed to $3.8 ; \xi_{1,2}$ are unit variance Gaussian distributed noise terms; and $\mu$ is set at 0.01 . We chose the start point $\left(x_{1}, y_{1}\right)$ randomly from $[0,1] \times[0,1]$ and generated time series of length 10000 with $\left(b_{1}, b_{2}\right) \in\{(0,0),(0,0.3),(0.1,0.3),(0.3,0.3)\}$. We repeated the same experiment with 100 different start points that are randomly chosen in $[0,1] \times[0,1]$. All these time series were not observed to diverge. The resulting directions of Granger causality were always the same. Fig. 4 shows Q-Q plots of p-values after 100 subsamples. In all 4 cases, our method identified the directions of coupling correctly: If $b_{1,2}=0$, both $\mathrm{X} \Rightarrow \mathrm{Y}$ and $\mathrm{Y} \Rightarrow \mathrm{X}$ are rejected due to uniform distributions; If $\left(b_{1}, b_{2}\right)=(0,0.3), \mathrm{X} \Rightarrow \mathrm{Y}$ is accepted and $\mathrm{Y} \Rightarrow \mathrm{X}$ gained non-evidence. In testing $\mathrm{Y} \Rightarrow \mathrm{X}$, we have due to the uc-area indirect evidence for the reversed direction, which is consistent with the generating model. In the case of $\left(b_{1}, b_{2}\right) \in\{(0.1,0.3),(0.3,0.3)\}$, both $\mathrm{X} \Rightarrow \mathrm{Y}$ and $\mathrm{Y} \Rightarrow \mathrm{X}$ are accepted. Interestingly, by means of kernel-based two-sample-test, we can additionally confirm that when $b_{1}=b_{2}=0.3$ the resulting p-values corresponding to testing $\mathrm{X} \Rightarrow \mathrm{Y}$ and to testing $\mathrm{Y} \Rightarrow \mathrm{X}$ are identically distributed, while in the case of $b_{1} \neq b_{2}$ the resulting $\mathrm{p}$-values corresponding to testing $\mathrm{X} \Rightarrow \mathrm{Y}$ and to testing $\mathrm{Y} \Rightarrow \mathrm{X}$ come from different distributions. This is reasonable, since the coupling is absolutely symmetric in $\mathrm{X}$ and $\mathrm{Y}$, if $b_{1}=b_{2}$. It seems plausible that the more right-skewed, the stronger the coupling (see the case $0.1=b_{1}<b_{2}=0.3$ ). But, we do not speculate on that.

To explore the limitation of the method, we conduct our test on data generated by highly noisy relations. Suppose $\mathrm{X}$ causes $\mathrm{Y}$ mediated by some hidden process $\mathrm{Z}$, i.e., $\mathrm{X} \Rightarrow \mathrm{Z} \Rightarrow \mathrm{Y}$. The uni-directed coupling between $X$ and $Z$, and between $Z$ and $Y$ are given by noisy logistic maps as in Eq. 5 with $b_{1}=0$. The coupling strength $b_{2}$ varies from 0.05 to 0.30 . The causal relation from $X$ to $Y$ is highly noisy and more sophisticated due to the indirect relation. We sampled time series of 10000 points from these models and tested the causal relation between $X$ and 

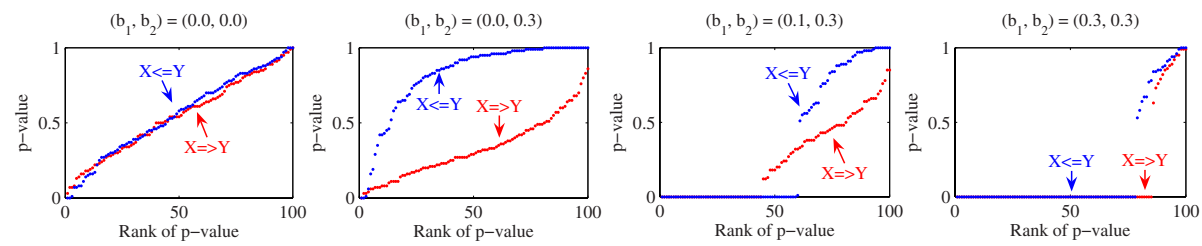

Fig. 4. Q-Q plots of p-values obtained by tests of predictability on 100 random subtime-series from time series generated by noisy logistic maps
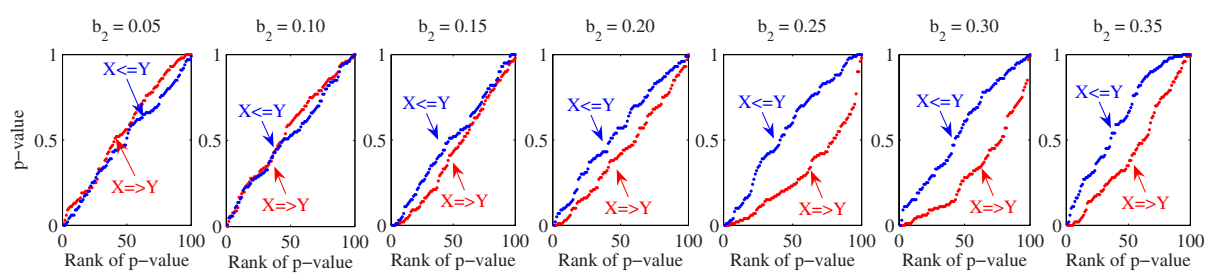

Fig. 5. Q-Q plots of p-values obtained by tests of predictability on 100 random subtime-series from time series of indirect relations generated by noisy logistic maps.

Table 1. Comparison of tests based on various causal measures (see text). The generating model is $\mathrm{X} \Rightarrow \mathrm{Y}$.

\begin{tabular}{|c||c|c|c|c|c|c|c||c|c|c|c|c|c|c|}
\hline \multicolumn{1}{|c||}{} & \multicolumn{9}{c||}{ Rejecting $\mathrm{X} \Leftarrow \mathrm{Y}$} & \multicolumn{6}{c|}{ Accepting $\mathrm{X} \Rightarrow \mathrm{Y}$} \\
\hline Coupling Strength $r$ & 0.05 & 0.10 & 0.15 & 0.20 & 0.25 & 0.30 & 0.35 & 0.05 & 0.10 & 0.15 & 0.20 & 0.25 & 0.30 & 0.35 \\
\hline \hline Kernel Test & 98 & 99 & 97 & 100 & 99 & 100 & 100 & 3 & 28 & 47 & 78 & 100 & 97 & 100 \\
\hline EGCI & 97 & 95 & 94 & 91 & 92 & 93 & 92 & 0 & 3 & 7 & 23 & 34 & 48 & 84 \\
\hline NLGC & 99 & 98 & 100 & 100 & 100 & 99 & 100 & 0 & 0 & 0 & 0 & 2 & 10 & 24 \\
\hline TE & 100 & 99 & 98 & 97 & 90 & 95 & 99 & 0 & 0 & 0 & 0 & 0 & 0 & 0 \\
\hline
\end{tabular}

$Y$ using different causality measures. Results for testing causal relation between $\mathrm{X}$ and $\mathrm{Y}$ on one sample is visualized in Fig. 5. In this sample, $\mathrm{X} \Leftarrow \mathrm{Y}$ is correctly rejected at different coupling strengths $b_{2} . \mathrm{X} \Rightarrow \mathrm{Y}$ is correctly accepted when the coupling strengths $b_{2}$ larger than 0.15 . Tab. 1 summarizes the results after 100 replications. In comparison, we conducted permutation tests based on the other causality measures, i.e., extended Granger causality index (EGCI) [6], nonlinear extensions of Granger causality (NLGC) [7, and Transfer entropy (TE) [24] (Significance level 0.05). The results show that all measures performance well in rejecting $\mathrm{X} \Leftarrow \mathrm{Y}$. In accepting $\mathrm{X} \Rightarrow \mathrm{Y}$, our kernel test performs better than EGCI. NLGC works bad, while TE fails completely. We conjecture that the reason that TE can not identify the relationship significantly is that the subsample size of 100 is too small for TE. After all, we expect that our kernel-based approach provides also a reasonable measure of Granger causality even in the case of small sample sizes. 


\subsection{Stock Price-Volume Relation}

We undertake an investigation of price and volume co-movement using the daily closing value DJ industrial average index and the daily volume of shares traded on NYSE from October 1928 to January 2008. The raw data are transformed to a series of day-to-day differences to obtain the daily movements (DM). The final dataset is a bivariate time series of 19920 observations (Fig. 6] left). Due to the large number of data points, we increase the number of subsamples to $N=$ 1000. The probability of the uniform distribution for the p-values corresponding to the hypothesis that the daily movement of price causes that of volume is $P^{\text {Unif }}=0<\frac{1}{2}$ ( $\mathrm{p}$-values are positively skewed); For the reserved causal direction, we have $P^{\text {Unif }}=0.940 \geq \frac{1}{2}$. Thus, we found strong evidence of asymmetry in the relationship between the daily movement of stock prices and the daily movement of trading volume. The daily movement of prices is much more important in predicting the movement of the trading volume, than the other way around.
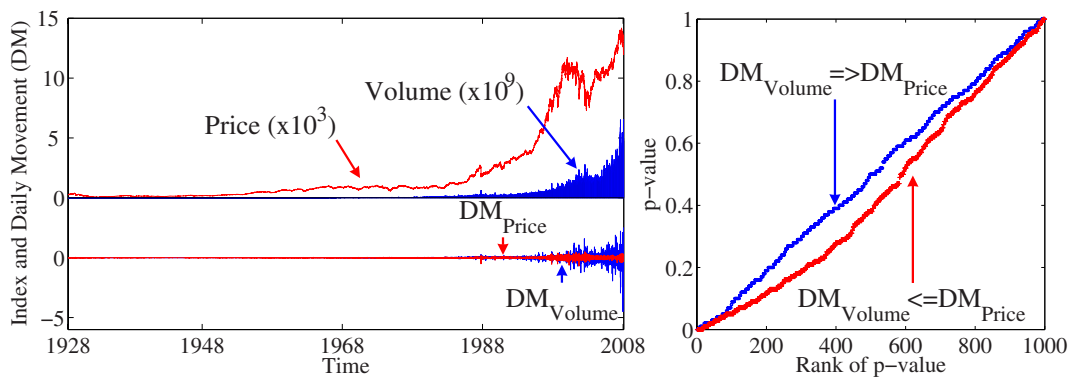

Fig. 6. DJ daily index, volume and movements (left). Q-Q plots of p-values (right) obtained by testing predictability between "DM$M_{\text {Volume" }}$ and " $\mathrm{DM}_{\text {Price }}$ " on 1000 random sub-time-series.

\subsection{Co-movement of Stock Indexes}

The analyzed raw dataset consists of daily closing values (adjusted for dividends and splits) of Dow Jones (DJ) industrial average index, Financial Times Stock Exchange (FTSE 100) and NIKKEI 225 stock average index during the time between April 1984 and January 2008. Only days with trading activity in both stock exchanges were considered. The time increment in the raw data is not always exactly one day due to weekends and moving holidays. We transform it into a series of day-to-day differences to describe the daily movements of indexes. After all, we have a trivariate time series with 5602 observations. We ran our test procedure on this dataset. The p-values obtained for all causal hypotheses are visualized in Fig. 7. The final testing results are summarized in Tab. 2 After all, our testing procedure showed evidence of following causality: DJ $\Rightarrow$ FTSE, 

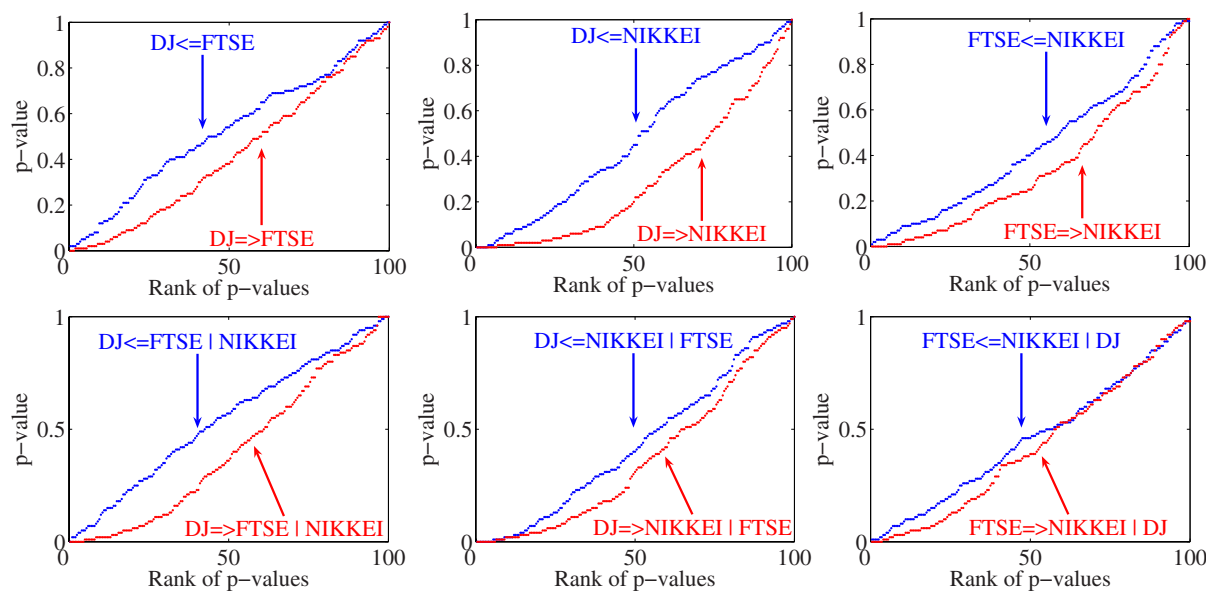

Fig. 7. Q-Q plots of p-values obtained by testing predictability between daily movements of DJ, FTSE and DJ on 100 random sub-time-series

Table 2. Testing results of causality between daily movements of DJ, FTSE and DJ on 100 random sub-time-series

\begin{tabular}{|c||c|c|c|}
\hline Causal Hypothesis & Skewness & $P^{\text {Unif }}$ & Testing Result \\
\hline DJ $\Rightarrow$ FTSE & positive & 0.70 & Accept \\
DJ $\Leftarrow$ FTSE & negative & 0.10 & Reject \\
\hline \hline DJ $\Rightarrow$ NIKKEI & positive & 1.00 & Accept \\
DJ $\Leftarrow$ NIKKEI & positive & 0.03 & Reject \\
\hline FTSE $\Rightarrow$ NIKKEI & positive & 1.00 & Accept \\
FTSE $\Leftarrow$ NIKKEI & positive & 0.65 & Accept \\
\hline \hline DJ $\Rightarrow$ FTSE $\mid$ NIKKEI & positive & 0.98 & Accept \\
DJ $\Leftarrow$ FTSE $\mid$ NIKKEI & negative & 0.13 & Reject \\
\hline DJ $\Rightarrow$ NIKKEI $\mid$ FTSE & positive & 1.00 & Accept \\
DJ $\Leftarrow$ NIKKEI $\mid$ FTSE & positive & 0.49 & Reject \\
\hline FTSE $\Rightarrow$ NIKKEI $\mid$ DJ & positive & 0.78 & Accept \\
FTSE $\Leftarrow$ NIKKEI $\mid$ DJ & positive & 0.08 & Reject \\
\hline
\end{tabular}

DJ $\Rightarrow$ NIKKEI and FTSE $\Leftrightarrow$ NIKKEI, whereas the causality running from the daily movement of NIKKEI to the daily movement of FTSE is spurious, since FTSE $\Leftarrow$ NIKKEI DJ is rejected. The knowledge of the dynamics of DJ can significantly improve a prediction of the dynamics of FTSE and NIKKEI, but the dynamics of FTSE and NIKKEI has a very limited, yet non-significant impact on the future dynamics of DJ. The finding that the movement of DJ influences the movement of FTSE and NIKKEI not vice versa, which may seem trivial as a purely economical fact, but actually confirms in an independent way the validity of the kernel test formalism. 


\subsection{Cardiorespiratory Interaction}

As the last example of real-life systems, we consider the benchmark bivariate time series of heart rate and respiration force of a sleeping human suffering from sleep apnea (data set B of the Santa Fe Institute time series competition [25]) recorded in the sleep laboratory of the Beth Israel Hospital in Boston, MA. The magnitudes considered are heart rate and respiration force. The data are plotted in Fig. 8 (left). The time interval between measurements is 0.5 seconds (sampling frequency $2 \mathrm{~Hz}$ ). As described in [26 27], under normal, physiological conditions, the heart rate is modulated by respiration through a process known as Respiratory Sinus Arrhythmia (RSA). It is the natural cycle of arrhythmia that occurs through the influence of breathing on the flow of sympathetic and vagus impulses to the sinoatrial node of the heart. When we inhale, vagus nerve activity is impeded and the heart rate begins to increase. When we exhale, this pattern is reversed. This quasi-periodic modulation of heart rate by respiration is most notable in young, healthy subjects and decreases with age, which means Heart $\Leftarrow$ Respiration. However, this dataset corresponds to a patient suffering from sleep apnea, which is a breathing disorder characterized by brief interruptions of breathing during sleep. Sleep apnea affects the normal process of RSA, disturbing the usual patterns of interaction between the heart rate and respiration. As a result, the control of the heart rate by respiration becomes unclear. It may well be blocked, in accordance with the change in dynamics, that is characteristic of the so-called "dynamical diseases". Some studies [72324 claimed a coupling in the reversed direction: Heart $\Rightarrow$ Respiration. In summary, the bidirected causation Heart $\Leftrightarrow$ Respiration might be likely the ground truth in this example. The result of our test procedure is consistent with this prior knowledge, since for both directions we have $P^{\text {Unif }}=0<\frac{1}{2}$ ( $\mathrm{p}$-values are positively skewed). Since the dynamics of this times series are rhythmic over time, we used embedding vectors with different $m, r$ as defined in Eq. 1. Only when $m \leq 20$ and $r \geq 12$, our test could not detect any significant causal relations between "Heart" and "Respiration", in other cases, a bidirected causality is always verified. The results
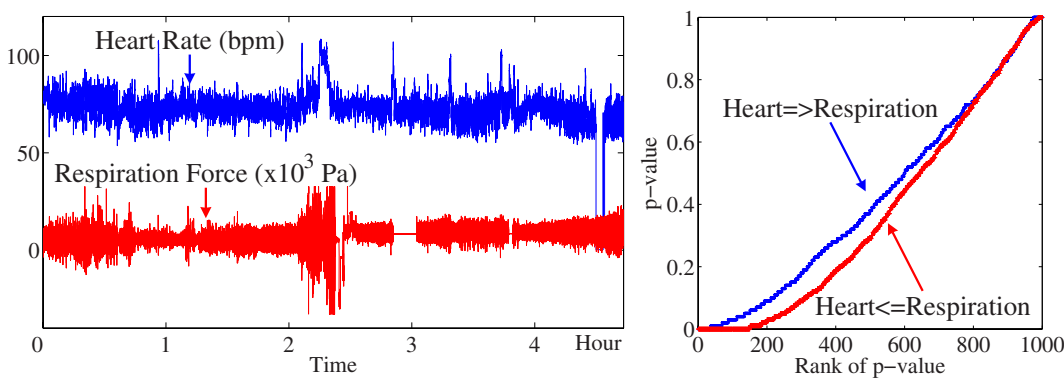

Fig. 8. Time series of the heart rate and respiration force of a patient suffering sleep apnea (left). Q-Q plots of p-values (right) obtained by testing predictability between "Heart" and "Respiration" on 1000 random sub-time-series. 
are plausible, because it is well known that the high-frequency band $(0.25-0.45$ $\mathrm{Hz})$ is characteristic of the respiratory rhythm. The identified bi-directed causation between heart rate and respiration suggests a probably causal link between sleep apnea and cardiovascular disease 28], although the exact mechanisms that underlie this relationship remain unresolved [29].

\section{Conclusion}

We have presented the kernel framework for detecting nonlinear Granger causality and have proposed to measure the improvement of the predictability by the trace norm of the conditional covariance operator and test whether the prediction error of the first time series can be significantly reduced by including knowledge from the second time series, given a third one. In comparison to other nonlinear extensions of Granger causality 6/7/15/24, our approach is designed in the general kernel framework and straightforwardly applicable. It is also appealing for users that the kernelized analysis of covariance is easy to implement and its statistical properties are well understood. Although kernel methods are rather popular nonlinear techniques in statistical pattern recognition, this is the first time, to the best of our knowledge, that the nonlinear extension of Granger's concept of causality in such a straightforward way is demonstrated.

Acknowledgement. Valuable discussions with Kenji Fukumizu are warmly acknowledged.

\section{References}

1. Packard, N.H., Crutchfield, J.P., Farmer, J.D., Shaw, R.S.: Geometry from a time series. Physical Review Letters 45, 712-716 (1980)

2. Takens, M.: Detecting strange attractors in turbulence. In: Rand, D.A., Young, L.S. (eds.) Dynamical Systems and Turbulence. Lecture Notes in Mathematics, pp. 366-381. Springer, Berlin (1982)

3. Kennel, M.B., Brown, R., Abarbanel, H.D.I.: Determining embedding dimension for phase-space reconstruction using a geometrical construction. Physical Review A 45(6), 3403-3411 (1992)

4. Fraser, A.M., Swinney, H.L.: Independent coordinates for strange attractors from mutual information. Physical Review A 33(2), 1134-1140 (1986)

5. Granger, C.: Investigating causal relations by econometric and cross-spectral methods. Econometrica 37, 424-438 (1969)

6. Chen, Y., Rangarajan, G., Feng, J., Ding, M.: Analyzing multiple nonlinear time series with extended Granger causality. Physics Letters A 324, 26-35 (2004)

7. Ancona, N., Marinazzo, D., Stramaglia, S.: Radial basis function approach to nonlinear granger causality of time series. Physical Review E 70, 66216.1-66216.7 (2004)

8. Aronszajn, N.: Theory of reproducing kernels. Transactions of the American Mathematical Society 68(3), 337-404 (1950)

9. Baker, C.: Joint measures and cross-covariance operators. Transactions of the American Mathematical Society 186, 273-289 (1973) 
10. Fukumizu, K., Bach, F., Jordan, M.: Kernel dimension reduction in regression. Technical Report 715, University of California, Berkeley, CA (2006)

11. Fukumizu, K., Gretton, A., Sun, X., Schölkopf, B.: Kernel measures of conditional dependence. In: Platt, J., Koller, D., Singer, Y., Roweis, S. (eds.) Proceedings of the 21th Neural Information Processing Systems Conference, pp. 489-496. MIT Press, Cambridge (2007)

12. Steinwart, I.: On the influence of the kernel on the consistency of support vector machines. Journal of Machine Learning Research 2, 67-93 (2001)

13. Sun, $\mathrm{X}$.: Causal inference from statistical data. $\mathrm{PhD}$ thesis, Computer Science Faculty, University of Karlsruhe (TH), Germany (2008)

14. Ancona, N., Stramaglia, S.: An invariance property of predictors in kernel-induced hypothesis spaces. Neural Computation 18, 749-759 (2006)

15. Marinazzo, D., Pellicoro, M., Stramaglia, S.: Nonlinear parametric model for Granger causality of time series. Physical Review E 73(6), 66216.1-66216.6 (2006)

16. Lukić, M., Beder, J.: Stochastic processes with sample paths in reproducing kernel Hilbert spaces. Transactions of the American Mathematical Society 353(10), 39453969 (2001)

17. Groetsch, C.: The theory of Tikhonov regularization for Fredholm equations of the first kind. Pitman Publishing Program, Boston (1984)

18. Hoerl, A., Kennard, R.: Ridge regression: Biased estimation for nonorthogonal problems. Technometrics 12(1), 55-67 (1970)

19. Fine, S., Scheinberg, K.: Efficient SVM training using low-rank kernel representations. Journal of Machine Learning Research 2, 243-264 (2001)

20. Hochberg, Y.: More powerful procedures for multiple significance testing. Statistics in Medicine 9(7), 811-818 (1990)

21. Schweder, T.: Plots of p-values to evaluate many tests simultaneously. Biometrika 69(3), 493-502 (1982)

22. Gretton, A., Borgwardt, K., Rasch, M., Schölkopf, B., et al.: A kernel method for the two-sample-problem. In: Schölkopf, B., Platt, J., Hoffman, T. (eds.) Proceedings of the 20th Neural Information Processing Systems Conference, pp. 513-520. MIT Press, Cambridge (2006)

23. Bhattacharya, J., Pereda, E., Petsche, H.: Effective detection of coupling in short and noisy bivariate data. IEEE Transactions on Systems, Man, and Cybernetics 33(1), 85-95 (2003)

24. Schreiber, T.: Measuring information transfer. Physical Review Letters 85(2), 461464 (2000)

25. Rigney, D., Goldberger, A., Ocasio, W., Ichimaru, Y., et al.: Multi-channel physiological data: description and analysis (data set B). In: Weigend, A., Gershenfeld, N. (eds.) Time series prediction: Forecasting the future and understanding the past, pp. 105-129. Addison-Wesley, Reading (1993)

26. Ichimaru, Y., Clark, K., Ringler, J., Weiss, W.: Effect of sleep stage on the relationship between respiration and heart rate variability. In: Proceedings of Computers in Cardiology 1990, pp. 657-660. IEEE Computer Society Press, Los Alamitos (1990)

27. Verdes, P.: Assessing causality from multivariate time series. Physical Review E 72(2), 066222.1-066222.9 (2005)

28. Roux, F., D'Ambrosio, C., Mohsenin, V.: Sleep-related breathing disorders and cardiovascular disease. The American Journal of Medicine 108(5), 396-402 (2000)

29. Duchna, H., Grote, L., Andreas, S., Schulz, R., et al.: Sleep-disordered breathing and cardio- and cerebrovascular diseases: 2003 update of clinical significance and future perspectives. Somnologie 7(3), 101-121 (2003) 